\title{
Future Challenges for Changes in the Disease Burden of Inpatients Living With HIV in China: a Predictive Study
}

\section{Yong Shuai ( $\square$ alexshuai@sina.com )}

Chongqing Public Health Medical Center https://orcid.org/0000-0002-1925-3011

Jingmin Nie

Chongqing Public Health Medical Center

\section{Yinqiu Huang}

Chongqing Public Health Medical Center

\section{Yaokai Chen}

Chongqing Public Health Medical Center

\section{Research}

Keywords: Inpatients living with HIV, AIDS-defining disease, Non-AIDS-defining disease, Prediction

Posted Date: May 10th, 2021

DOl: https://doi.org/10.21203/rs.3.rs-459948/v1

License: @ (i) This work is licensed under a Creative Commons Attribution 4.0 International License. Read Full License 


\section{Future challenges for Changes in the disease burden of inpatients living with HIV in China: a predictive study}

Yong Shuai ${ }^{1,2}$, Jingmin $\mathrm{Nie}^{1}$, Yinqiu Huang ${ }^{1}$, Yaokai Chen ${ }^{1}$

\section{Summary}

Objective:In China, the widespread use of Combination Antiretroviral Therapy(cART) has significantly improved the prognosis and quality of life in people living with HIV(PLWH). However, some PLWH still got worse and were hospitalized for some reasons, including AIDS-Defining Diseases(AD, such as opportunistic infections and AIDS-related Malignancies), Non-AIDS-Defining Diseases (NAD, such as non-AIDS-defining Malignancies and metabolic syndrome). At present, the age and disease of inpatients living with HIV(ILWH) are undergoing some significant changes. For example, the average age of ILWH is gradually increasing, the proportions of ILWH with NAD in all age groups are increasing, and the proportion of ILWH suffering from multiple types of NAD is increasing, which will have a greater impact to both ILWH and infectious disease hospitals. We aim to predict the changes in ILWH in the next 10 years, analyze the impact of such changes on ILWH and infectious disease hospitals in China, and propose corresponding countermeasures.

Methods:We have established a prediction model based on Artificial Neural Network and Time series, using 12618 data of ILWH admitted to Chongqing Public Health Medical Center during 2010-2020, to predict the age of ILWH, the proportion of $\mathrm{AD}$ and $\mathrm{NAD}$, the proportion of multiple types of NAD, and the proportion of NAD occurrences among various age groups from 2021 to 2030 .

Results:Our model shows that the average age of ILWH has risen from 43.8 years in 2010 to 57.6 years in 2030 , and the increase in the age of women is greater than that of men. The number of ILWH in different age groups has changed significantly. Among them, the proportion of ILWH under 50 years old has gradually decreased, and the proportion of ILWH over 50 years old has gradually raised. By 2030, the proportion of inpatients over 50 years old will be close to $80 \%$. The proportion of fatal opportunistic infections among ILWH has decreased year by year, and the most common diseases are tuberculosis-related diseases. The proportions of ILWH with only AD or NAD have gradually decreased, and the proportion of ILWH with both AD and NAD has increased year by year. It is estimated that the proportion of all ILWH with both AD and NAD will exceed $80 \%$ by 2030 . Among all kinds of diseases in NAD, metabolic syndrome will have the highest rate of increase. By 2030, it will account for $16 \%$ in all ILWH. The proportion of hepatobiliary and pancreatic system diseases and blood system diseases in NAD will gradually decrease, while the proportion of metabolic syndrome, kidney disease, gastrointestinal disease, non-AIDS defining malignancies(NADM), bone disease and neurological diseases in NAD will gradually increase. The proportion of ILWH with NAD in all age groups will increase significantly after 10 years. Among them, the proportion of ILWH with NAD in 50-60 years old will be more than $80 \%$, and among the ILWH under 30 years old, the proportion of ILWH suffering from NAD will increase to $69.6 \%$. The proportion of ILWH without NAD or only 1 NAD will decrease, while the proportion of ILWH with 3 or more NAD will rise sharply. The proportion of ILWH with 1 type of NAD will drop to $12.6 \%$, while the proportion of ILWH with 2 types of NAD, and the proportion of ILWH with 3 or more types of NAD will increase greatly.

Conclusions:Significant changes have took place in the disease burden of ILWH in China, including the aging, the increase in the proportion of ILWH with NAD, and the increase in the proportion of ILWH with multiple types of NAD. These changes mean that in the next 10 years, the diagnosis, treatment and care of ILWH, as well as the development and construction of infectious disease hospitals will all face new challenges. This finding is based on a large number of long-term ILWH data in Chongqing, China, so we can consider that our research strategy can be promoted in China to improve the cure rate and quality of life in ILWH.

Keywords: Inpatients living with HIV, AIDS-defining disease, Non-AIDS-defining disease, Prediction

\section{Introduction}

*Correspondence: alexshuai@sina.com; yaokaichen@ hotmail.com

$\dagger$ Yong Shuai and Jingmin Nie contributed equally to this work

1 Chongqing Public Health Medical Center, Chongqing, 400035, China

2 College of Pharmacy, Southwest University, Chongqing, 400700 China 
In recent years, due to the widespread application of cART and the continuous expansion of HIV prevention and education coverage, the rapid rise trend of the AIDS epidemic in China has slowed down and the mortality rate has declined, but the situation remains grim. By the end of 2020, China CDC data shows that the number of reported survive people living with HIV is 1,053,000. However, as some HIV-infected people do not know they have been infected with HIV (about 32.1\% of HIV-infected people in China do not know their infection status), are lack of good compliance, have poor immune reconstitution, or have received organ transplants, their immunity is generally low and are prone to opportunistic infections or AIDS-related malignancies, and they need to be hospitalized for treatment and care. [1-3] At the same time, with the increase of the survival rate in people living with HIV ( PLWH), PLWH are facing with some new problems such as ,premature aging[4-7], increasing in biological age[8-11], frailty [12,13], multiple comorbidities[14-18]. Because of the serious condition, some patients also need to be hospitalized for treatment and care .

The current analysis and research on HIV-infected populations usually target all HIV-infected populations in some certain areas[19]. The research contents mainly focuses on the mortality[20-22], the incidence of certain AD[20,23] and NAD[14-18,24-31], the mechanism[32] and strategies[7,33] of the aging and frailty in PLWH, but there is no comprehensive analysis and predictive research on ILWH.

Therefore, we constructed a combined time series forecasting model. By predicting the changes of ILWH in the next 10 years, we found the changes in the age and disease burden of ILWH, analyze the impact of the change on the diagnosis and treatment, nursing, management of ILWH and the construction and development of infectious disease hospitals in China, and discuss corresponding countermeasures.

\section{Methods}

Definitions

$\mathrm{AD}$ refers to the diseases that cause various serious opportunistic infections and malignancies due to the body has been infected by HIV. AD diseases mainly include opportunistic infections and AIDS-defining malignancies . AD mainly include Bacterial infections, multiple or recurrent; Candidiasis of bronchi, trachea, or lungs; Candidiasis of the esophagus; Cervical cancer (invasive); Coccidioidomycosis, disseminated; Cryptococcosis, presenting outside of the lung; Cryptosporidiosis, chronic intestinal for longer than one month; Cytomegalovirus (CMV) with loss of vision; Cytomegalovirus disease (other than in the liver, spleen, or lymph nodes); Encephalopathy (HIV-related, also knows as AIDS dementia complex); Herpes simplex virus (HSV), lasting longer than a month or appearing in an area other than the skin (such as esophagus or lungs); Histoplasmosis, disseminated; Kaposi's sarcoma (KS); Lymphoid interstitial pneumonia or pulmonary lymphoid hyperplasia complex; Burkitt lymphoma (or equivalent term); Immunoblastic lymphoma (or equivalent term); Primary lymphoma of the brain; Mycobacterium avium complex or Mycobacterium kansasii; Mycobacterium tuberculosis of any site in or out of the lungs; Mycobacterium or similar species, disseminated beyond the lung; Pneumocystis pneumonia caused by the fungus Pneumocystis jiroveci+Pneumonia, recurrent; Progressive multifocal leukoencephalopathy (PML); Salmonella septicemia, recurrent; Toxoplasmosis of the brain; Tuberculosis; Wasting syndrome; Non-Hodgkin's lymphoma, and other infections caused by human immunodeficiency virus[34,35].

Non-AIDS-defining diseases are diseases that are not caused by HIV. Based on the disease spectrum research and natural language extraction from the database in this paper, we have summarized 18 types of NAD that ILWH suffer from, including metabolic syndrome, cardiovascular and cerebrovascular diseases, kidney diseases, hepatobiliary and pancreatic system diseases, digestive tract diseases, non-AIDS-defining malignancies(NADM), bone diseases, inflammatory autoimmune diseases, respiratory diseases, ENT diseases, eye diseases, and mental diseases , Nervous system disease, skin disease, venereal disease, urinary system disease, blood system disease, lymphatic system disease, accidental injury.

\section{Data source}

The data comes from the Chongqing Public Health Medical Center, the only tertiary infectious disease hospital in Chongqing. From January 2010 to December 2020, Chongqing Public Health Medical Center has received 17,150 
person-years data on ILWH.

The data collected includes the gender, age, length of hospital stay, outpatient diagnosis information, admission diagnosis information, and discharge diagnosis information in ILWH.

The data inclusion criteria are: (1) Patients with confirmed HIV infection. (2) First-time hospitalized patients (to prevent certain chronic diseases from being counted many times). (3) The hospitalization time is not less than 1 day (to prevent the inclusion of data of patients who have not met the hospitalization conditions).

Taking into account the needs of the forecasting model, the data exclusion criteria are:

(1) Inpatients who are not infected with HIV.

(2) Patients with abnormal data, such as the error caused by the negative value of the patient's age.

(3) There is no detailed disease data record. Such as the patient's case only recorded as "abdominal pain", "white blood cell abnormality", and the disease can not be classified.

(4) In order to ensure the credibility of the prediction model, it is necessary to delete these disease categories which are less than $1 \%$ of the total number of diseases.

Based on the data exclusion criteria, 12 categories of NAD were retained in the study, including metabolic syndrome, cardiovascular and cerebrovascular diseases, kidney diseases, hepatobiliary and pancreatic system diseases, gastrointestinal diseases, NADM, bone disease, respiratory system disease, nervous system disease, skin disease and venereal disease, blood system disease, lymphatic system disease.

According to the data inclusion and exclusion criteria, there are finally 12618 person-year data available, and the data are independent of each other. The basic information of the data set is shown in Table 1.

\section{Data calculation instructions}

Limited by the total number of hospital beds, the overall number of ILWH will not change much. Therefore, we consider to discuss the proportion of different patients with AD or NAD in the total number of ILWH .

The calculation methods of various values are as follows:

1. The proportion of $\mathrm{AD}(\mathrm{NAD})$ patients = the total number of $\mathrm{AD}(\mathrm{NAD})$ patients in the year / the total number of ILWH in the year * $100 \%$.

2. Proportion of patients with various diseases $=$ total number of patients with this type of disease in the year/ the total number of ILWH in the year * $100 \%$.

3. Age group statistics refer to literature [7], which divided the groups into six time periods, including less than 30 , 30-40, 40-50, 50-60, 60-70, and more than 70 .

\section{Model}

We have established a prediction model based on Artificial Neural Network and time series model. The model first uses natural language processing technology based on artificial neural networks to preprocess the disease spectrum, and extracts the disease spectrum corresponding to the database text. Then we use the improved Seasonal AutoRegressive Moving Average (SARIMA) model to establish a time series model and predict the disease development in the next ten years. The model can dynamically predict the trend of data changes over time, and ensure that the overall disease incidence prediction value of the patient is consistent with the incidence prediction value of various types of diseases, ensuring that the prediction results have good consistency and credibility .

\section{Results}

Through model prediction, we mainly observe the prediction results from the following eight aspects.

\section{Statistical analysis of the age of ILWH}

From 2010 to 2030, the average age and median of ILWH will increase significantly, and the average value will rise from 43.8 years to 57.6 years, an increase of about 14 years. At the same time, compared with male, the age of female ILWH has increased more rapidly, from 41 to 66 years.

\section{Age group analysis of ILWH}

From 2010 to 2030, the proportion of ILWH in all ages will change significantly. Among them, the proportion of 
ILWH under 50 years old will gradually decrease, and the proportion of ILWH over 50 years old will gradually increase. By 2030, the proportion of ILWH under 30 years old will be less than $10 \%$, and the proportion of ILWH over 50 years old will exceed $80 \%$. Among them, ILWH aged 50-60 will account for the largest proportion, reaching nearly $40 \%$.

3. Changes in some fatal opportunistic infections in ILWH

Disease spectrum studies have shown that cryptococcal meningitis [36], pneumocystis pneumonia(PCP) [37], toxoplasma encephalopathy [38], cytomegalovirus retinitis [39], cyanobacteria marneffei [40] and Tuberculosis [41] are the most dangerous types of highly fatal opportunistic infections, accounting for more than $90 \%$ of hospitalized AIDS cases and deaths in China. Therefore, we studied the changes in the proportion of ILWH with these six fatal diseases.

Through the prediction model, it is found that the total proportion of fatal opportunistic infections in ILWH will declined year by year, and it will decrease from $64 \%$ at the peak in 2016 to about $45 \%$ in 2030 . At the same time, among the patients with fatal opportunistic infections, tuberculosis is the most common infections, and the proportion of PCP, toxoplasma encephalitis, cytomegalovirus retinitis, cyanobacteria marneffei and cryptococcal meningitis will gradually decrease.

\section{Changes in ILWH with only $\mathrm{AD}$, only NAD, and both $\mathrm{AD}$ and NAD}

It can be seen from the figure that the proportion of ILWH with only AD and only NAD will gradually decrease and be less than $10 \%$ by 2030; The proportion of ILWH with both AD and NAD will increase year by year and exceed $80 \%$ in 2030 .

\section{Changes in various NAD in ILWH}

In the next ten years, the proportion of ILWH with NAD will increase, and its proportion will rise from $59.5 \%$ in 2010 to $79.8 \%$ in 2030 , an increase of more than $20 \%$, and nearly $80 \%$ of ILWH will Suffer from NAD. Among them, the metabolic syndrome accounted for the highest proportion of all NAD, which will account for $16 \%$ of all ILWH in 2030, and be close to one-sixth of the total. Among all NAD, the proportion of metabolic syndrome, kidney disease, gastrointestinal diseases, NADM, bone diseases and neurological diseases will have a large increase, while hepatobiliary and pancreatic system diseases and hematological will decrease, and other diseases in NAD will remain roughly the same.

6. Changes in the proportion of ILWH with NAD in various age groups

The proportion of ILWH with NAD in all age groups will increase significantly in the next 10 years. The proportion of ILWH with NAD in the 50-60 years will be the fastest growing age group. By 2030, the proportion of ILWH with NAD aged 50-60 will exceed 80\%. Among ILWH under the age of 30, the proportion of ILWH suffering from NAD will rise from $62.4 \%$ to $69.6 \%$; among ILWH aged 30-40, the proportion of ILWH with NAD will rise from $60.5 \%$ to $62.3 \%$, indicating that HIV-infected people with NAD also tend to be younger, and HIV-infected people are more likely to age and suffer from senile diseases than normal people. This situation is similar to the research content of literature [8].

7. Proportion of ILWH with multiple NAD

The ratio of ILWH without NAD and ILWH with 1 NAD will decrease in the next ten years, and the proportion of ILWH with 2 NAD will increase slightly, ILWH with 3 or more NAD will rise sharply, and the proportion will rise to about $45 \%$ by 2030 .

In the results of this study, patients without NAD, patients with $2 \mathrm{NAD}$, and patients with 3 or more NAD have the same trend as the literature [7], but trend of patients with one NAD is different from literature [7]. In literature 7, the number of patients with one NAD is also gradually increasing, but this article found that the proportion of ILWH with one NAD is decreasing, which may be caused by the difference in the groups studied.

\section{Proportion of ILWH with multiple types of NAD}

The research on NAD in this paper is mainly divided into 12 types of diseases. What we studied in this section is the proportion of patients suffering from one category to multiple categories, which is different from the section above. If a patient suffers from diabetes and hypertension at the same time, because both diseases belong to the metabolic 
syndrome, the patient is defined as having one type of NAD. If a patient suffers from hyperlipidemia and osteoporosis at the same time, because this patient suffers from metabolic syndrome and bone disease, this patient is defined as having two types of NAD.

Based on the prediction model, it can be found that the proportion of patients with one type of NAD will decrease from $37.5 \%$ to $12.6 \%$, and the proportion of patients with two types of NAD will rise from $15.6 \%$ to $22.2 \%$, the proportion of patients with 3 or more types of NAD will increase from $3.6 \%$ to $45 \%$, indicating that the proportion of patients infected with multiple types of NAD will increase significantly in the next ten years .

\section{Discussion}

From the above results, we can find that some research results of ILWH are similar to those of general HIV-infected people, including aging [4-7], premature aging [8-11], the number of patients suffering from NAD is gradually increasing [7,14-18], and the number of patients with multiple NAD is increasing year by year [7].

At the same time, some new research results only appeared in ILWH, and there is no literature to verify these phenomena for the time being. Therefore, we need to discuss these results.

1.The proportion of ILWH with both AD and NAD will increase year by year.

There may be three reasons to explain this result. Firstly, because most of ILWH are in the AIDS stage, their age is larger than that of the general HIV infected patients, so they are prone to opportunistic infections, and they are also prone to have NAD which is easy for the elderly. [42] Secondly, most of ILWH were hospitalized because of their serious condition. These patients are prone to suffer from AD and NAD at the same time because of their poor immunity [43]. Thirdly, due to the increase in the proportion of medical insurance reimbursements and the increase in the disposable income of the people in China in recent years, as well as the increase in the awareness of PLWH to seek medical treatment, most PLWH are willing to receive better treatment and care through hospitalization. These reasons have led to the increase in the proportion of patients with both $\mathrm{AD}$ and NAD, and have also created new challenges for the diagnosis, treatment and service capabilities of infectious disease hospitals.

2. The proportion of patients with fatal opportunistic infections is decreasing year by year, and TB is the fatal opportunistic infections disease with the highest proportion.

As China has expanded the scope of HIV testing for people at high risk of AIDS and the general population, many HIV-infected people can be detected early in HIV infection period and receive antiretroviral treatment, so that HIV-infected people will not progress to the AIDS stage, and reduce the probability of opportunistic infections. At the same time, with the continuous improvement of China's opportunistic infection prevention and control strategies (Including primary and secondary prevention(control) measures for opportunistic infections) for HIV-infected patients [36-41], it is reasonable that the proportion of fatal opportunistic infections in China has been declining year by year.

The reason why tuberculosis is the disease with the highest proportion is mainly because of the huge number of tuberculosis infections (disease savings pool) in China, which is expected to reach 350 million. These tuberculosis-infected people are likely to transmit Mycobacterium tuberculosis to HIV-infected people with low immunity. At the same time, when the body's immunity declines, the immune system cannot control Mycobacterium tuberculosis nodules, and the pathogen will attack the body and spread to other organs. The incidence of this infection being activated is about $5-10 \%$ during the lifetime of latent tuberculosis infection (LTBI). Because the immunity of HIV-infected people is destroyed by HIV, their immunity is usually very poor, so they are more likely to activate LTBI.

3. Among all NAD, the proportion of metabolic syndrome has increased year by year, and has maintained the largest proportion. The increase in the proportion of ILWH with metabolic syndrome may be related to factors such as aging, the effects of antiretroviral drugs, chronic inflammation caused by HIV, and changes in the lifestyle of the Chinese population.

(1)From the results of this study, it can be seen that the aging of HIV hospitalized patients is more serious. The decline of body function due to aging increases the risk of ILWH suffering from metabolic syndrome.

(2)Antiretroviral drugs (such as integrase inhibitor Kelizhi [45]) may affect the metabolism of triacylglycerols in 
the body, leading to excessive accumulation of triacylglycerols in the body. The accumulation of triacylglycerol in liver cells can cause non-alcoholic fatty liver, the accumulation in muscle and pancreatic $\beta$ cells may lead to insulin resistance and diabetes, and insulin resistance can cause hyperinsulinemia. Indinavir, a protease inhibitor in antiviral drugs, can cause insulin resistance and trigger hyperglycemia, while nucleoside drugs can cause insulin resistance through indirect effects such as fat redistribution.

(3) The impact of HIV. HIV can cause chronic inflammation in the body of an infected person. Chronic inflammation may lead to an increase in the amount of epicardial fat, and may lead to an increase in coronary calcium, myocardial perfusion defects and myocardial infarction. At the same time, HIV also affects pancreatic $\beta$-cell function and insulin secretion.[46]

(4)Due to changes in Chinese lifestyles, the average prevalence of overweight and obesity has risen from $5.3 \%$ to $20.5 \%$ [47], and obesity may be one of the causes of metabolic syndrome [48,49]. According to statistics, among the top ten causes of death among Chinese residents, more than one-third of the diseases are related to metabolic syndrome[50], this may also be one of the reasons for the increase in the proportion of metabolic syndrome among ILWH.

4. Among the non-AIDS-defined diseases, hepatobiliary and pancreatic system diseases and blood system diseases account for a gradual decrease in the proportion of NAD. At the same time, the proportions of metabolic syndrome, kidney disease, gastrointestinal disease, non-AIDS defined malignancies, bone disease and neurological disease are gradually increasing. The reasons for the changes in the proportion of metabolic syndrome have been explained above, and the changes in other diseases will be discussed next.

(1)The liver is the main metabolic site of most drugs used to treat opportunistic infections. The liver damage in the early years was mainly caused by opportunistic infection diseases related drugs. With the gradual decrease in the proportion of PLWH with opportunistic infections, and the continuous improvement of drugs for the treatment of opportunistic infections, the damage to the liver is gradually reduced, resulting in a continuous decline in the proportion of ILWH with hepatobiliary and pancreatic diseases. [51,52]

(2)The blood system disease spectrum established in this paper shows that the main blood diseases include anemia, pancytopenia, thrombocytopenia, agranulocytosis, and neutropenia. Most of these blood diseases are caused by HIV and are related to the physique of HIV-infected persons. As China has increased its efforts to screen for HIV, HIV-infected persons will be detected and treated in the early stages of infection. At the same time, most patients will pay attention to enhancing the body's nutrition and improving the body's immunity after knowing that they are infected with HIV. The above two reasons have led to a reduction in the incidence of blood system diseases.

(3) The increase in the proportion of kidney disease, gastrointestinal disease, neurological disease, and NADM may be related to immunodeficiency, the direct role of HIV-1, antiretroviral therapy toxicity, viral co-infections, behavioral risk factors, inflammation and ageing. Because the aging and low immunity of ILWH are more common, they are more susceptible to the above six factors and suffer from these NAD than ordinary HIV infected people. Many papers have demonstrated these reasons[17,19,53-61]. Studies have shown that NADM is the main cause of death among HIV-infected persons with NAD[7,17,22,35], so early screening of HIV-inpatients is needed.

(4) Bone diseases include bone damage caused by external forces, as well as pathological bone diseases. The reasons for the increase in the proportion of bone diseases can be summarized in four points. Firstly, the effects of some antiretroviral drugs lead to changes in bone metabolism (such as tenofovir), which makes HIV-infected people suffer from bone diseases [62]. Secondly, because the immune system mediates a powerful effect on bone turnover, when the HIV virus destroys the human immune system, the HIV virus affects bone mass, causing osteoporosis or bone loss, thereby inducing the risk of fractures. [63,64]. Thirdly, due to the increasing aging in ILWH, the elderly have aging physical functions, weakened physical coordination and control capabilities, and are likely to cause bone damage due to falls. [65] Fourthly, as Chinese infectious disease hospitals have attached great importance to the bone mineral density examination of HIV-infected persons in recent years, most hospitals have purchased more advanced bone density testing equipment and discovered some unobvious bone diseases, which may also be the cause of the increase in bone diseases.

5. The proportion of ILWH with multiple types of NAD is increasing year by year 
The human body is an extremely complex and precise structure. The various systems of the human body are connected, restricted and coordinated with each other to maintain the normal progress of various complex life activities inside and outside the human body and ensure the continuation of life. Due to the multiple effects of various systems of the human body, HIV and anti-HIV drugs, the body functions of ILWH may undergo some chain changes, resulting in ILWH suffering from multiple types of NAD.

If a certain system has dysfunction, it will affect the functions of other organs. For example, the kidney secretes 1,25-dihydroxyvitamin D3, which regulates calcium metabolism. Therefore, patients with chronic kidney disease may also suffer from renal bone disease; Decreased renal excretion function will increase cardiovascular load and cause cardiac insufficiency; Renal anemia further increases the burden of cardiovascular function and affects heart function; When patients with nephrotic syndrome have a hypercoagulable state of hypoproteinemia, they may be complicated by cardiovascular and cerebrovascular embolism and other cardiovascular and cerebrovascular diseases. Cardiac dysfunction affects pulmonary circulation, leading to pulmonary dysfunction and renal insufficiency. Therefore, when there is a problem with a certain system in an ILWH, they may suffer from more types of NAD due to their weaker body functions and immunity. [33]

\section{Description of the data credibility}

Since the prognostic data, medication data(Including antiretroviral therapy data and other and comorbidity therapy data), and medical inspection data(Including CD4+ T lymphocyte count, HIV RNA viral load, liver and kidney function testing, etc.) in the database collected by the paper are not complete, we did not use these data in the prediction model, and only established the analysis and prediction model based on the ILWH case text data. This may have a certain impact on the prediction results of the model. However, we have verified that the prediction results of the model are credible through comparison with relevant literature, as well as adjustments and accuracy tests of the model.

\section{Strategy}

In response to the above-mentioned newly discovered problems, we put forward some strategies to the infectious disease hospital and ILWH. The purpose is to improve the diagnosis, treatment and nursing capacity of infectious disease hospitals for ILWH, improve the survival rate and life quality of ILWH, and promote China to achieve the "90-90-90" goals early.

1. The construction of the infectious disease hospital. In general, the main role of infectious disease hospitals is to treat patients with various infectious diseases and achieve the overall management and control of infectious diseases. However, as the changes of inpatients' condition, the construction direction of infectious disease hospitals also needs to be adjusted.

(1) In terms of talent construction in infectious disease hospitals, it is necessary to introduce diverse talents to achieve coverage for treatment of multiple diseases. At present, the diagnosis and treatment of patients in infectious disease hospitals are usually undertaken by infectious disease doctors. Due to the lack of professional knowledge of NAD diagnosis and treatment, infectious disease doctors are often unable to perform comprehensive diagnosis and treatment of NAD, such as performing heart surgery, liver and kidney surgery, and interventional surgery. Therefore, it is necessary to improve the personnel construction system of infectious disease hospitals.

(2) In terms of the discipline construction of infectious disease hospitals, it is necessary to establish departments(Including general surgery, dermatology, obstetrics and gynecology, ophthalmology, otorhinolaryngology, orthopedics, endocrinology, cardiovascular and cerebrovascular departments, etc.) that patients need as soon as possible, improve the diagnosis and treatment capabilities of the departments, and achieve comprehensive protection for patients.

(3) The application of Multi-disciplinary treatment mode (MTM). [66] MTM can provide patients with the most effective individualized treatment plan with the least adverse reactions and the best quality of life. Using MTM to establish a relatively fixed expert team composed of experts in related disciplines can solve the diagnosis and treatment problems of patients infected with multiple types of NAD.

(4)Establish isolation wards for opportunistic infections and non-opportunistic infections. As hospitalized patients infected with HIV are generally severely ill and have weakened immunity, they are susceptible to opportunistic 
infections. Therefore, patients with opportunistic infections and those with non-opportunistic infections should be managed in separate wards to reduce the risk of cross-infection.

(5)Introduce advanced auxiliary diagnosis and treatment technologies (such as auxiliary diagnosis and treatment technology based on big data and artificial intelligence, medical impact recognition technology) and information systems (such as remote consultation system, electronic diagnosis and treatment system) to assist in the diagnosis and treatment of ILWH. Especially for remote areas and areas with underdeveloped medical resources, the use of information and intelligent methods to assist inexperienced doctors in the diagnosis and treatment of patients can increase the utilization of medical resources, reduce the rate of misdiagnosis, and ultimately improve the survival rate and life quality of ILWH.

2. Diagnosis and treatment, nursing, management, prevention and scientific research for ILWH.

(1) In terms of diagnosis and treatment to ILWH, it is necessary to scientifically and rationally plan a multi-drug combination treatment plan for the aging patients and patients with multiple types of NAD.

(2)In terms of nursing ILWH, the hospitals should establish a theoretical system similar to elderly care, and to train relevant skills of medical staff to realize scientific nursing of HIV inpatients.

(3) In terms of management, the hospitals should establish an intelligent full-process management mode to realize the full-process management of HIV-infected persons. Use the follow-up process to assist patients to improve their lifestyles, including quitting smoking, reducing saturated fatty acid intake, scientific diet, aerobic exercise, and so on.

(4) Prevention strategies of ILWH mainly include the prevention strategies of AD and NAD. For the prevention of $\mathrm{AD}, \mathrm{HIV}$-infected persons should receive antiretroviral treatment as soon as possible, actively enhance their own immunity, reduce contact with the source of infection, cut off the route of transmission, and carry out preventive treatment of opportunistic infections. For the prevention of NAD, the main purpose is to cultivate a good medical awareness of patients, develop the habit of regular inspections, and implement early inspections, treatments and early control of NAD. Especially the patients who were infected with HIV during young and middle-aged period should pay more attention to the prevention of premature aging and NAD.

(5)In terms of scientific research, in order to improve the life quality of HIV-infected people, it is necessary to study the mechanisms of HIV-infected aging, premature aging, and increased incidence of NAD [10,29,31,43,67,68], study the changes in body functions of elderly HIV-infected persons (such as physiological functions, vital organs functions), develop relevant drugs and study drug absorption, adverse reactions, and pharmacokinetics, and provide personalized medication plans for HIV-infected people of all age range and suffering from various comorbidities.

\section{Conclusion}

In the next ten years, the disease burden of ILWH in China will continue to increase and will undergo major changes, including aging, premature aging, suffering from multiple and multiple types of NAD, and so on. These changes make ILWH and infectious disease hospitals face new challenges. In response to these challenges, we have proposed corresponding plans, which is conducive to improving the cure rate and life quality of ILWH, and is applicable to other regions in China. In the future, we will improve the NAD disease spectrum, collect complete data on ILWH, including prognostic data, medication data, and inspection data, and analyze and predict these data from multiple perspectives.

\section{Declarations}

\section{Consent for publication}

Written informed consent for publication was obtained from all participants.

\section{Availability of data and materials}

The datasets used or analyzed during the current study are owned by Chongqing Public Health Medical Center. Since the data includes sensitive patient data and involves some patents under development, the data can not be shared.

\section{Ethics approval and consent to participate}

The study complies with the principles of the Declaration of Helsinki and was approved by the Human Science 
Ethics Committee of Chongqing Public Health Medical Center. The Human Science Ethics Committee authorized the waiver of informed consent based on the observational nature of this study, and the ethics review approval document number: 2021-005-01-KY. The clinical information data was extracted from the patient comprehensive management main database, and it was studied anonymously.

\section{Competing interests}

The authors declare that they have no financial conflicts of interest.

\section{Funding}

This study was supported by the Chongqing Science and Technology Bureau Project ( cstc2019jscx-fxyd0298, cstc2020jscx-cylh0004).

\section{Authors' contributions}

JMN, YQH collected and provided the data. YS extracted data and cleaned data. YS designed the study, constructed the model, and analyzed the data. YKC and JMN provided the correlative Knowledge. YS drafted of the manuscript. All authors read and approved the final manuscript.

\section{Acknowledgements}

We would like to express our gratitude to all participants involved in this study and the staff of Chongqing Public Health Medical Center.

\section{Authors' information}

The corresponding author is a postdoctoral fellow, focusing on the interdisciplinary research of artificial intelligence and AIDS.

\section{References}

[1] Maschke, Matthias. Opportunistic infections in patients with HIV. Nervenheilkunde, 2020; 39(9); 536-541

[2] Dufaur L., Matin N.. Important opportunistic infections in HIV(Review). Medicine (United Kingdom), 2018; 46(6); 352-355

[3] Eduardo Perez-Alba, Itzel Araceli Ortiz-Meza, Luis Ángel Ceceñas-Falcón, Adrián Camacho-Ortiz. Multiple opportunistic infections in a patient with HIV infection. Clinical Obstetrics \& Gynecology, 2019; 62(4), 1-5

[4] Giovanni Guaraldi, Jovana Milic, Cristina Mussini. Aging With HIV. Current HIV/AIDS reports, 2019 ; 16 (6); 475-481

[5] Giovanni Guaraldi, Frank J. Palella Jr. Clinical implications of aging with HIV infection: perspectives and the future medical care agenda. AIDS, 2017; 31(2); 129-135

[6] A. Calcagno,S. Nozza, C. Muss, B. M. Celesia, et al. Ageing with HIV: a multidisciplinary review. Infection, 2015; 43(5); 509-522

[7] Mikaela Smit, Kees Brinkman, Suzanne Geerlings, Colette Smit, et al. Future challenges for clinical care of an ageing population infected with HIV: a modelling study. LANCET INFECTIOUS DISEASES , 2015; 15(9); 810-818

[8] Andrew M. Gross, Philipp A. Jaeger, Jason F. Kreisberg, Katherine Licon, et al. Methylome-wide Analysis of Chronic HIV Infection Reveals Five-Year Increase in Biological Age and Epigenetic Targeting of HLA. Molecular Cell. 2016; 62(2); 157-168

[9] Konstantinos Boulias;Judy Lieberman;Eric Lieberman Greer;An Epigenetic Clock Measures Accelerated Aging in Treated HIV Infection. Molecular Cell, 2016; 62(2); 153-155

[10] Gross, Andrew M., (Jaeger, Philipp A., Kreisberg, Jason F., Licon, Katherine, et al. Methylome-wide Analysis of Chronic HIV Infection Reveals Five-Year Increase in Biological Age and Epigenetic Targeting of HLA. Molecular Cell, 2016; 62(2); 157-168

[11] Erlandson, Kristine M.;Karris, Maile Y.. HIV and Aging. Infectious Disease Clinics of North America, 2019; 33(3); 769-786

[12] Sharma Anjali, Shi Qiuhu, Hoover Donald R.,Tien, Phyllis C, et al. Frailty predicts fractures among women with 
and at-risk for HIV. AIDS. 2019; 33(3); 455-463

[13] A Beanland, J Alagaratnam, C Goffe, A Bailey, et al. Objective and subjective rapid frailty screening tools in people with HIV. HIV Medicine. 2020; 22(2); 146-150

[14] Yinzhong Shen, Jiangrong Wang, Zhenyan Wang,Tangkai Qi, et al. Prevalence of Dyslipidemia Among Antiretroviral-Naive HIV-Infected Individuals in China. Medicine. 2015; 94(48); 1-8

[15] Yinzhong Shen, Zhenyan Wang, Li Liu, Renfang Zhang, et al. Prevalence of hyperglycemia among adults with newly diagnosed HIV/AIDS in China. BMC Infectious Diseases, 2013; 13(79); 1-6

[16] PeiYing Wu, ChienChing Hung, WenChun Liu, ChiaYin Hsieh. Metabolic syndrome among HIV-infected Taiwanese patients in the era of highly active antiretroviral therapy: prevalence and associated factors. Journal of Antimicrobial Chemotherapy, 2012; 67(4); 1001-1009

[17] Marco Franzetti, Elena Ricci, Paolo Bonfanti. The Pattern of Non-AIDS-defining Cancers in the HIV Population: Epidemiology, Risk Factors and Prognosis. A Review. Current HIV Research, 2019; 17(1); 1-12

[18] Delphine Lebrun, Maxime Hentzien, Lise Cuzin, David Rey. Epidemiology of autoimmune and inflammatory diseases in a French nationwide HIV cohort. AIDS, 2017; 31(15); 2159-2166

[19] Shiels MS, Islam JY, Rosenberg PS1, Hall HI, Jacobson E, Engels EA. Projected cancer incidence rates and burden of incident cancer cases in HIV-infected adults in the United States through 2030. Annals of Internal Medicine, 2018; 168(12); 866-873

[20] Fred Stephen Sarfo, Maame Anima Sarfo, Betty Norman, Richard Phillips, et al. Risk of Deaths, AIDS-Defining and Non-AIDS Defining Events among Ghanaians on Long-Term Combination Antiretroviral Therapy. PLOS One, 2014; 10(9); 1-13

[21] April C Pettit, Mark J Giganti, Suzanne M Ingle, Margaret T May, et al. Increased non-AIDS mortality among persons with AIDS-defining events after antiretroviral therapy initiation. Journal of the International AIDS Society, 2017; 21(1); 1-8

[22] Colette J Smith, Lene Ryom, Rainer Weber, Philippe Morlat, et al. Trends in underlying causes of death in people with HIV from 1999 to 2011 (D:A:D): a multicohort collaboration. Lancet , 2014 ;384(9939); 241-248

[23] Yunxuan Huang, Oulu Zhou, Zhigang Zheng, Yuexiang Xu, et al. Effect of AIDS-defining events at initiation of antiretroviral therapy on long-term mortality of HIV/AIDS patients in Southwestern China: a retrospective cohort study. AIDS Research and Therapy, 2020; 17(44); 1-9

[24] Cristina Lpez, Mar Masi, Sergio Padilla, Ana Aquilino, Cristina Bas, Flix Gutirrez. Deaths due to non-AIDS diseases among HIV infected patients: A 14-year study (1998-2011). Enfermedades infecciosasy microbiologia clinica ,2016; 34(4); 222-227

[25] Gentilotti, Elisa;Santoro, Maria M;Dori, Luca;Maffongelli et al. Impact of HIV-1 tropism on the emergence of non-AIDS events in HIV-infected patients receiving fully suppressive antiretroviral therapy. AIDS, 2016; 30(5); 731-741

[26] S Zhang;A van Sighem;A Kesselring;L Gras, et al. Risk of non-AIDS-defining events among HIV-infected patients not yet on antiretroviral therapy. HIV medicine, 2015; 16(5); 265-272

[27] Yu Wang, Hongyuan Liang, Ling Zhang, Zhe Zhang, et al. The burden of serious non-AIDS-defining events among admitted cART-naive AIDS patients in China: An observational cohort study. PloS one, 2020; 15(12); 1-17

[28] Worm Signe Westring, De Wit Stéphane, Weber Rainer, Sabin Caroline, et al. Diabetes Mellitus, Preexisting Coronary Heart Disease, and the Risk of Subsequent Coronary Heart Disease Events in Patients Infected With Human Immunodeficiency Virus The Data Collection on Adverse Events of Anti-HIV Drugs. CIRCULATION, 2009; 119(6); 805-811.

[29] Y.M. Pacheco, I. Jarrin, I. Rosado, A.A. Campins, et al. Increased risk of non-AIDS-related events in HIV subjects with persistent low CD4 counts despite cART in the CoRIS cohort. Antiviral Research, 2015; 117; 69-74

[30] C. William Wester, John R. Koethe, Bryan E. Shepherd, Samuel E. Stinnette, et al. Non-AIDS-defining events among HIV-1-infected adults receiving combination antiretroviral therapy in resource-replete versus 
resource-limited urban setting. AIDS , 2011; 25(12); 1471-1479

[31] Cristina Mussini, Patrizia Lorenzini, Alessandro Cozzi-Lepri, Giuseppe Lapadula, et al. CD4/CD8 ratio normalisation and non-AIDS-related events in individuals with HIV who achieve viral load suppression with antiretroviral therapy: an observational cohort study. The lancet. HIV, 2015; 2(3); 98-106

[32] Weiwei Cao, Beth D. Jamieson, Lance E. Hultin, Patricia M. Hultin, Rita B. Effros, Roger Detels, Roger. Premature Aging of T cells Is Associated With Faster HIV-1 Disease Progression, Journal of Acquired Immune Deficiency Syndromes. 2009; 50(2); 137-147

[33] Steven G. Deeks, Julie Overbaugh, Andrew Phillips, Susan Buchbinder. HIV infection. Nature reviews disease primers, 2015; $1 ; 1-22$

[34] Centers for Disease Control and Prevention (CDC). Revised surveillance case definition for HIV infection-United States, 2014. MMWR Recomm Rep. 2014; 63(03); 1-10

[35] Mark Bower, A Palfreeman, Maryam Alfa-Wali, Chris Bunker. British HIV Association Guidelines for HIV-associated Malignancies 2014. HIV MEDICINE, 2014;15(2); 1-92

[36] Chen Yaokai, Wu Hao. Expert Consensus on Diagnosis and Treatment of Cryptococcosis in AIDS Patients, Journal of Southwest University(Natural Science Edition), 2020.6; 42(7); 1-19

[37] Chen Yaokai, Wu Hao. Expert Consensus on Diagnosis and Treatment of Pneumocystis Pneumonia in AIDS Patients in China. Journal of Southwest University(Natural Science Edition), 2020.6; 42(7); 49-60

[38] Chen Yaokai, Wu Hao. Expert Consensus on Diagnosis and Treatment of Toxoplasmic Encephalitis in AIDS Patients. Journal of Southwest University(Natural Science Edition), 2020.7; 42(7)); 38-48

[39] Chen Yaokai, Wu Hao. Expert Consensus on Diagnosis and Treatment of Cytomegalovirus Disease in AIDS Patients. Journal of Southwest University(Natural Science Edition), 2020.7; 42(7); 20-37

[40] Chen Yaokai, Wu Hao. Expert Consensus on Diagnosis and Treatment of Tlromyrchosis in AIDS Patientsin China. Journal of Southwest University(Natural Science Edition), 2020.7; 42(7); 61-75

[41] Shen Yinzhong, Lu Hongzhou. Expert consensus on diagnosis and treatment of HIV infection combined with Mycobacterium tuberculosis infection. Chinese Journal of clinical infectious diseases, 2017; 10(2); 81-90

[42] Lu Hongzhou. Commonly used drugs and interactions for AIDS and related diseases. Shanghai Science and Technology Press. 2020.1; 1-10

[43] A. Mocroft, H. J. Furrer, J. M. Miro, P. Reiss, et al. The Incidence of AIDS-Defining Illnesses at a Current CD4 Count $\geq 200$ Cells $/ \mu \mathrm{L}$ in the Post-Combination Antiretroviral Therapy Era. Clinical Infectious Diseases, 2013; 57(7); 1038-1047

[44] Emilia Harding. WHO global progress report on tuberculosis elimination. The Lancet Respiratory Medicine, 2020; $8(1) ; 1-208$

[45] Dai Lili, Ye Jiangzhu, Shao Ying; Li Jianwei, Ma Hongli, Sun Lijun Effect of antiviral therapy regimen based on Kaletra on blood lipid: a retrospective clinical study in adult patients with HIV/AIDS. CHINESE Journal of AIDS \& STD, 2017; 23(9); 806-809

[46] Lu Hongzhou, Shen Yinzhong. Diagnosis and Treatment Atlas of AIDS and Related Diseases. Shanghai Science and Technology Press. 2019.6; 119-121

[47] Yanhui Dong;Catherine Jan;Yinghua Ma;Bin Dong, et .al. E Economic development and the nutritional status of Chinese school-aged children and adolescents from 1995 to 2014: an analysis of five successive national surveys. , The lancet. Diabetes \& endocrinology. 2019; 7(4); 288-299

[48] Longenecker, Chris T;Buggey, Jonathan. Heart fat in HIV: marker or mediator of risk?. Current opinion in HIV and AIDS. 2017; 12(6); 572-578

[49] Carl J Lavie 1, Abhishek Sharma 2, Martin A Alpert 3, Alban De Schutter, et al. Update on Obesity and Obesity Paradox in Heart Failure. Prog Cardiovasc Dis,2016; 58(4); 393-400.

[50] Maigeng Zhou;Haidong Wang;Xinying Zeng;Peng Yin, et al. Mortality, morbidity, and risk factors in China and its provinces, 1990-2017: a systematic analysis for the Global Burden of Disease Study 2017. Lancet, 2019; 
394(10204); 1145-1158

[51] Bouglouga O.a, Bagny A.b, Lawson-Ananissoh L.M.b, Yakoubou R.E.-H.b, et al. Hepatobiliary diseases in people with HIV infection at the Lome, Togo, university hospital: Epidemiologic and diagnostic aspect. Medecine et Sante Tropicales, 2018; 28(2); 193-196

[52] Sherman KE, Peters MG, Thomas DL. HIV and the liver. Topics In Antiviral Medicine, 2019;27(3); 101-110

[53] Hughes Kelly, Chang Jerry, Stadtler Hannah, Wyatt, Christin, et al. HIV-1 infection of the kidney: mechanisms and implications. AIDS, 2020.3; 35(3); 359-367

[54] Christina M Wyatt. Kidney Disease and HIV Infection. Topics in antiviral medicinee, 2017; 25(1); $13-16$

[55] Saraladevi Naicker. HIV/AIDS and Chronic Kidney Disease. Clinical nephrology, 2020; 93(1); 87-93

[56] Miro J.M., Cofan F., Trullas J.C., Manzardo C., et al. Renal dysfunction in the setting of HIV/AIDS. Current HIV/AIDS Reports, 2012; 9(3); 187-199

[57] Jae H Sim, Shibani S Mukerji, Samuel C Russo, Janet Lo . Gastrointestinal Dysfunction and HIV Comorbidities. Current HIV/AIDS reports, 2021; 18(1); 57-62

[58] Brew, Bruce J.a,b,c;Barnes, Stephanie L.a . The impact of HIV central nervous system persistence on pathogenesis. AIDS, $2019 ; 33(2) ; 113-121$

[59] Victoria Rojas-Celis, Fernando Valiente-Echeverría, Ricardo Soto-Rifo, Daniela Toro-Ascuy . New Challenges of HIV-1 Infection: How HIV-1 Attacks and Resides in the Central Nervous System. Cells. 2019; 8(10); 1245-1245

[60] Rebecca T Veenhuis, Janice E Clements, Lucio Gama. HIV Eradication Strategies: Implications for the Central Nervous System. Current HIV/AIDS reports, 2019; 16(1); 96-104

[61] M Ceccarelli;E Venanzi Rullo;M A Marino;F d'Aleo, et al. Non-AIDS defining cancers: a comprehensive update on diagnosis and management. European review for medical and pharmacological sciences, 2020; 24(7); 3849-3875

[62] WANG Jufeng,XU Lijun,HUANG Ying,XIE Yimi,et al.Impact of long-term highly active antiretroviral therapy on bone metabolism in AIDS patients. Journal Of Zhejiang University (Medical Sciences), 2016;45(3); 228-235

[63] M. Neale Weitzmann. Bone and the Immune System. Bone Toxicology, 2017; 45(7); 911-924

[64] Schumacher B.HIV infection also goes to the bones. MMW-Fortschritte der Medizin. 2019; 161(20); 26

[65] LYU Wei,WANG Ou,LI Tai-sheng. Recommendations on management of bone diseases in human immunodeficiency virus-infected patients. Clinical Medication Journal, 2021.3;19(3); 1-8

[66] WU Jun, XU Lie, SHEN Yinzhong. Implementation and effectiveness of the multi-disciplinary treatment mode for AIDS. Shanghai Medical \& Pharmaceutical Journal, 2020; 41(11); 21-23

[67] Mocroft,A., Furrer,H.J., Miro,J.M., Reiss,P., et al. The incidence of AIDS-defining illnesses at a current CD4 count $\geq 200$ cells $/ \mu \mathrm{L}$ in the post-combination antiretroviral therapy era. Clinical infectious diseases, 2013; 57(7); $1038-1047$

[68] Martin Hoenigl, Carlee B. Moser, Nicholas Funderburg, Ronald Bosch, et al. Soluble Urokinase Plasminogen Activator Receptor Is Predictive of Non-AIDS Events During Antiretroviral Therapy-mediated Viral Suppression. Clinical Infectious Diseases, 2019; 69(4); 676-686 
Figures

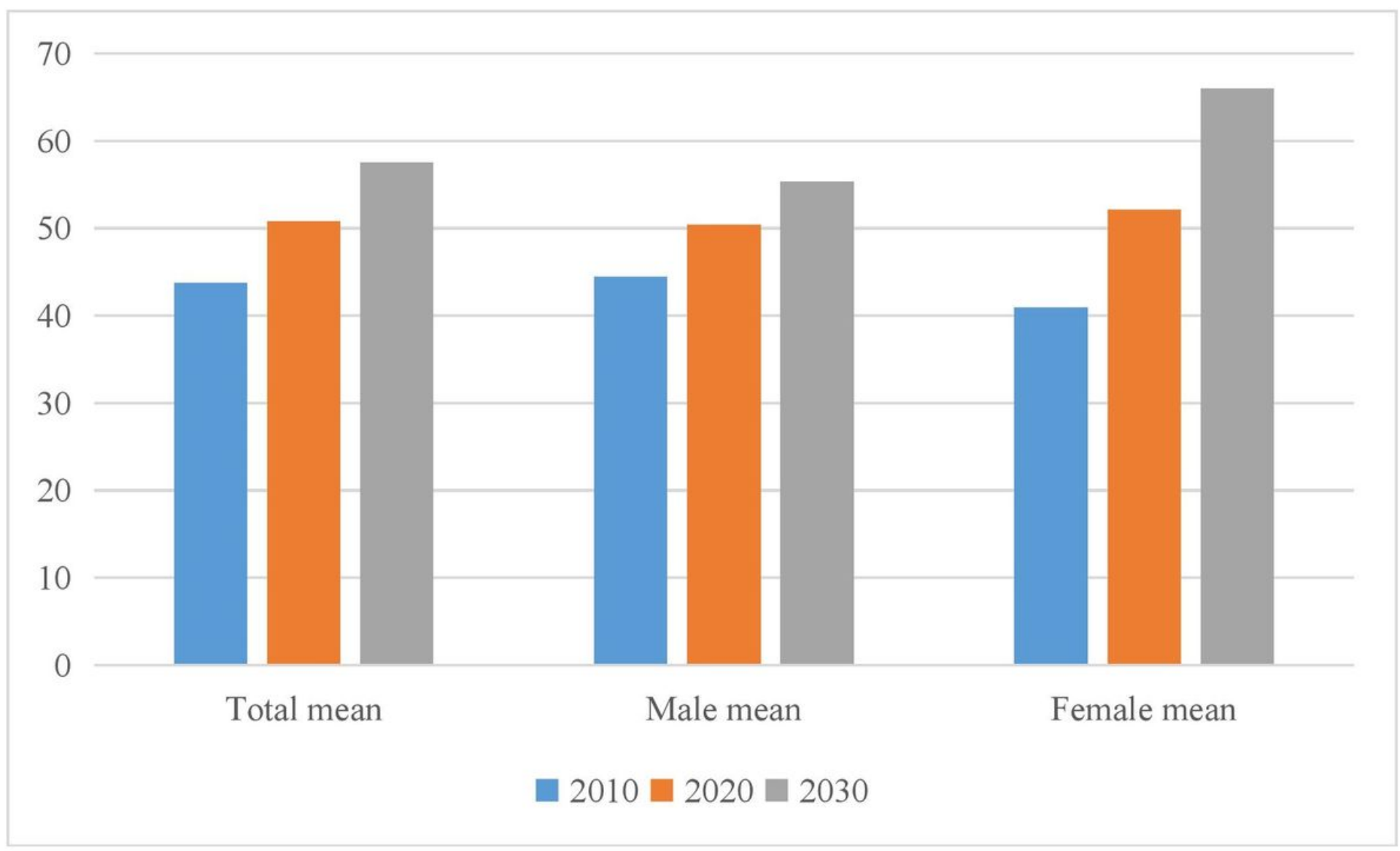

Figure 1

Age statistics of ILWH 


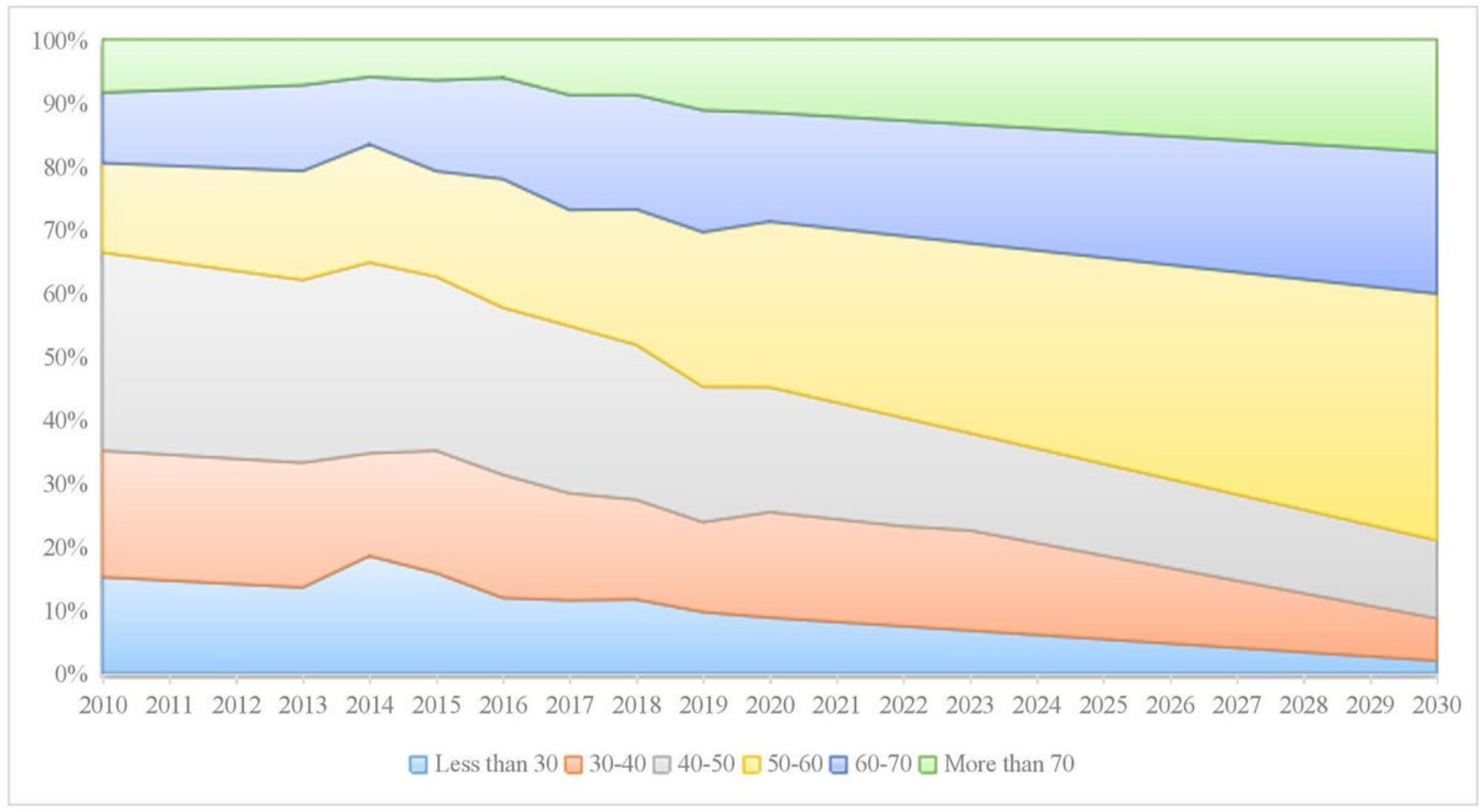

Figure 2

Proportion of all age groups in ILWH

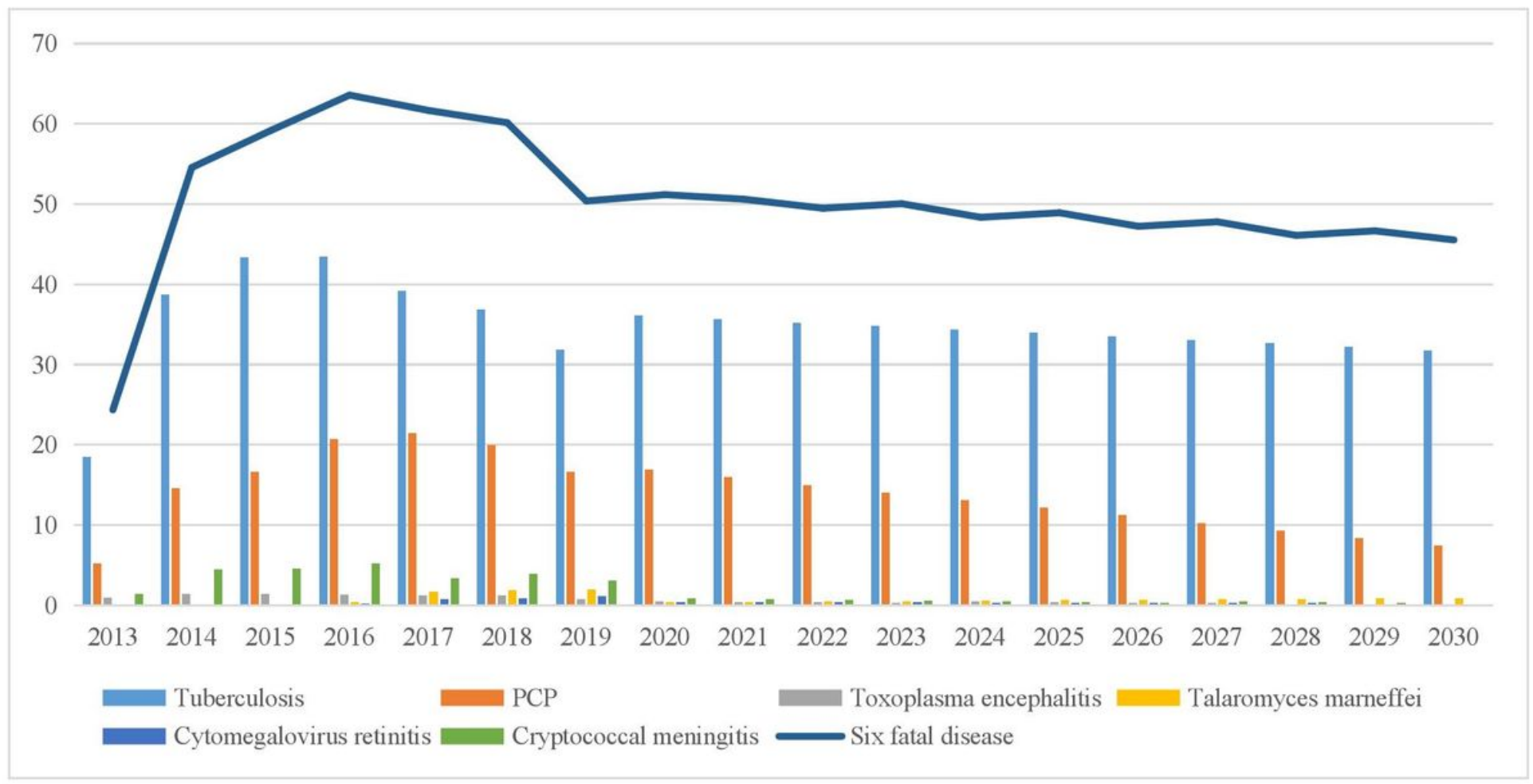

Figure 3 
Changes in some fatal opportunistic infections in ILWH

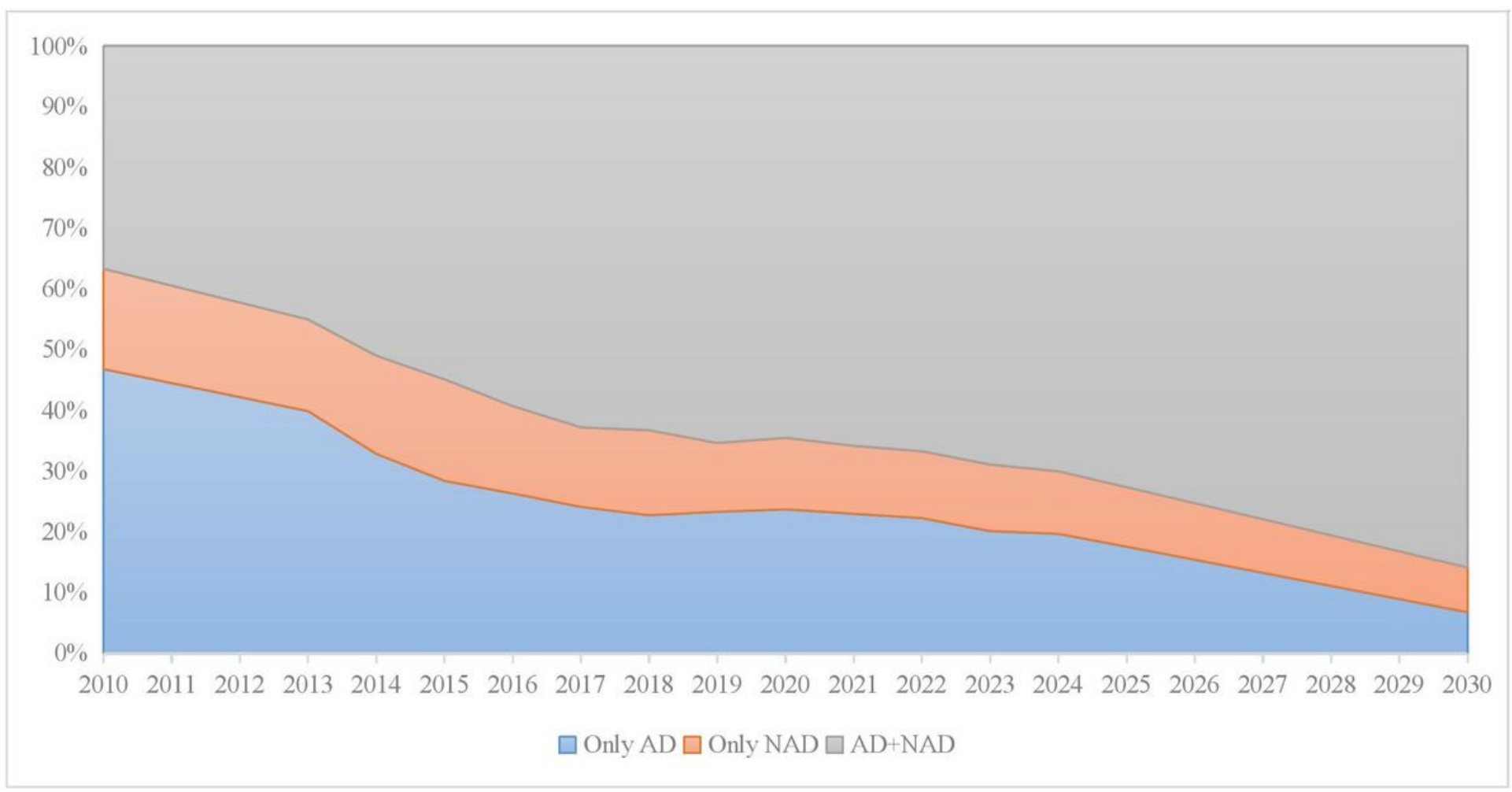

\section{Figure 4}

Changes in ILWH with only AD, only NAD, and both $A D$ and NAD

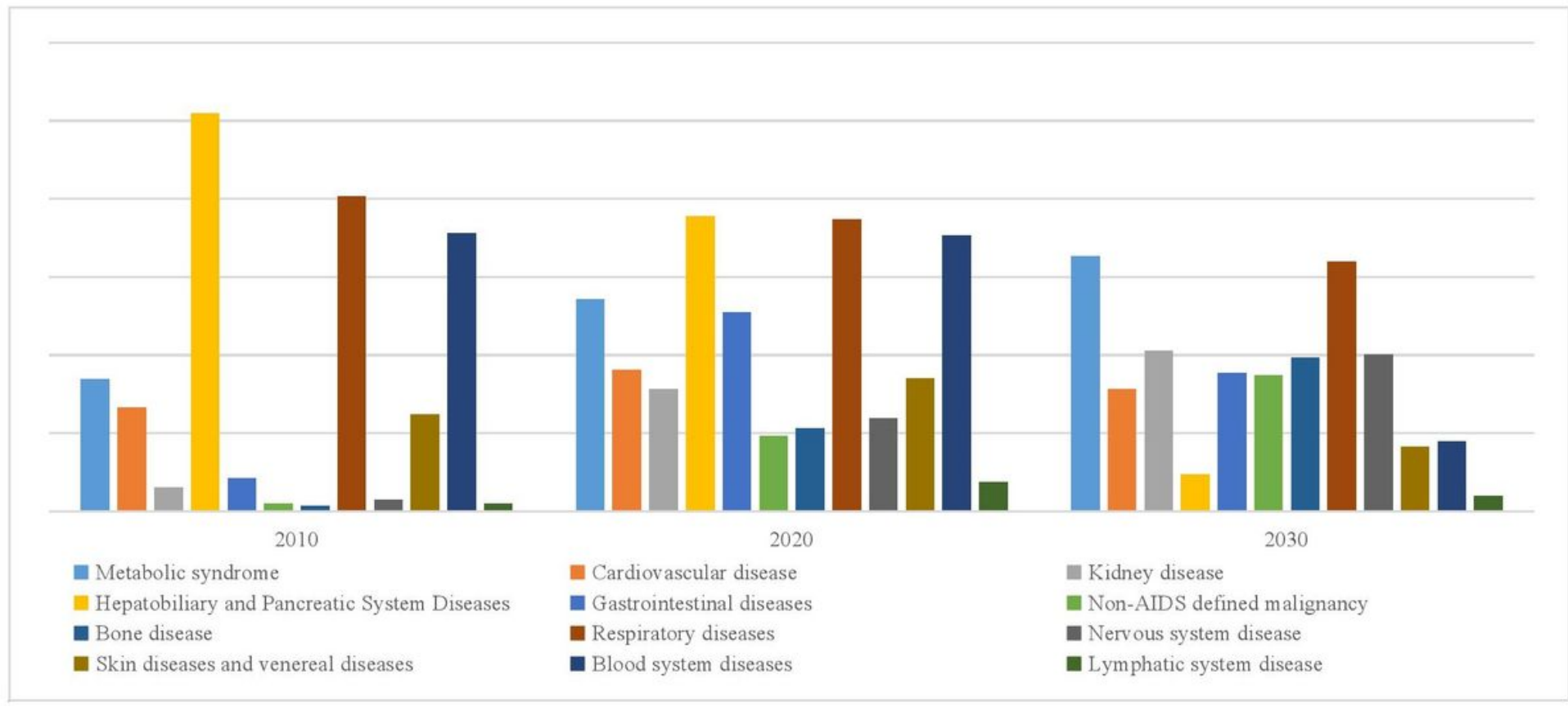

\section{Figure 5}

Ratio change of various NAD in ILWH 


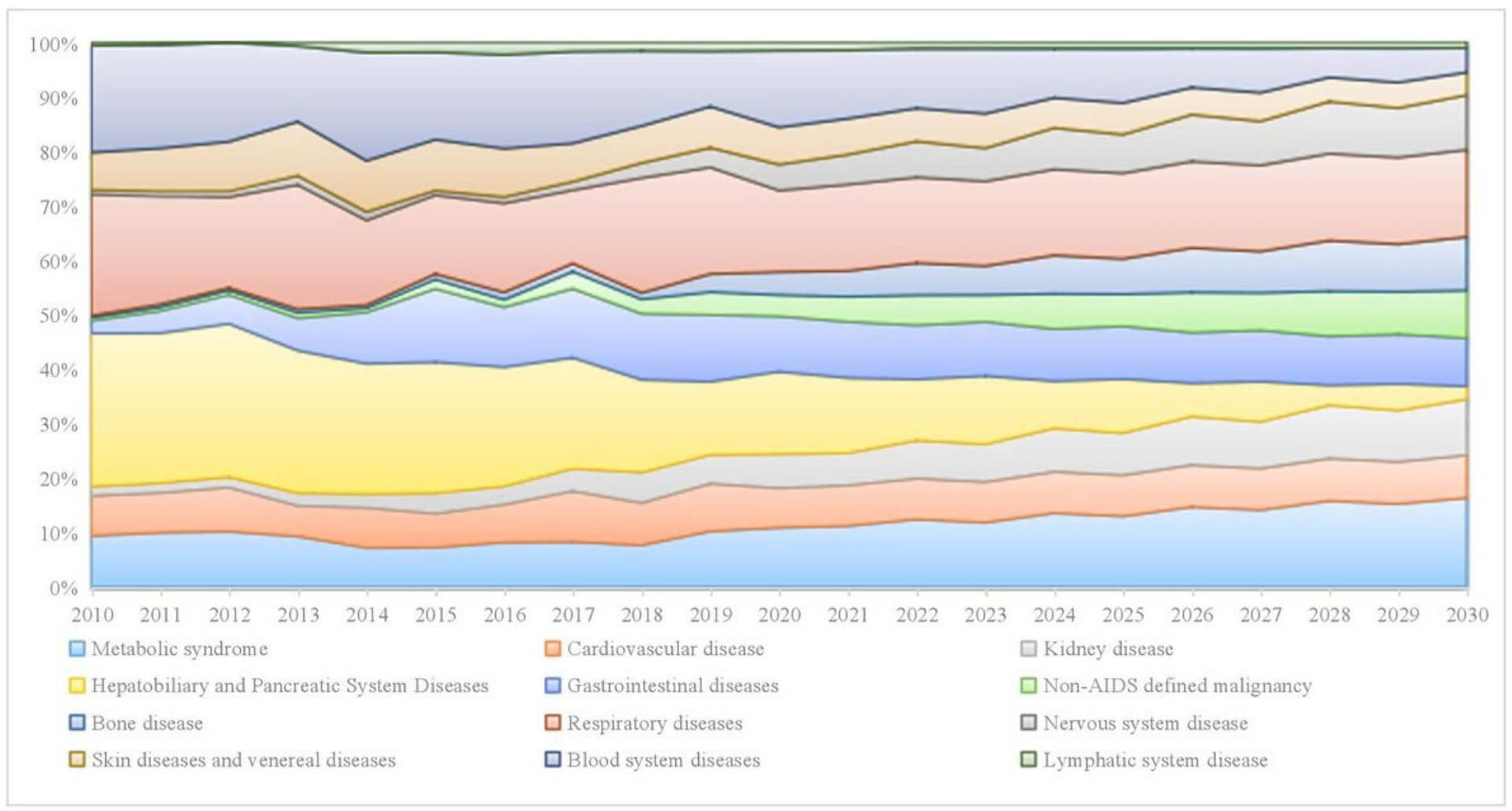

Figure 6

Proportion change of various NAD in ILWH

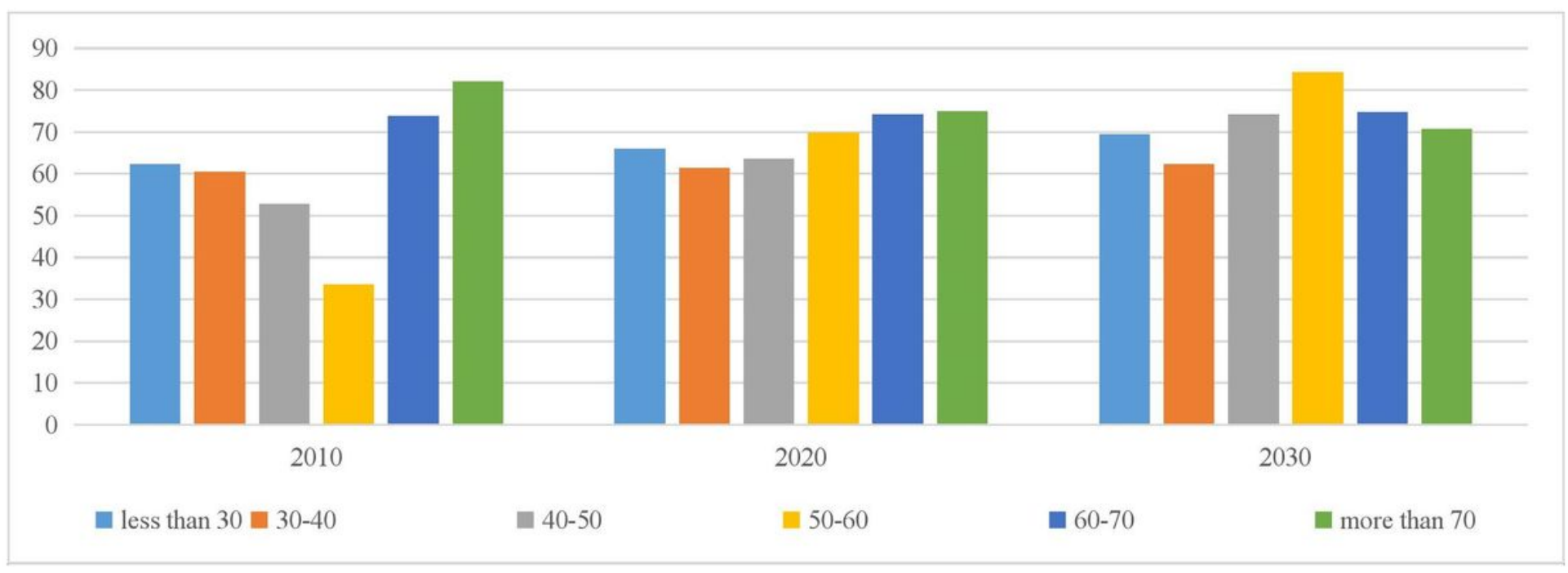

Figure 7

Changes in the proportion of ILWH with NAD in various age groups 


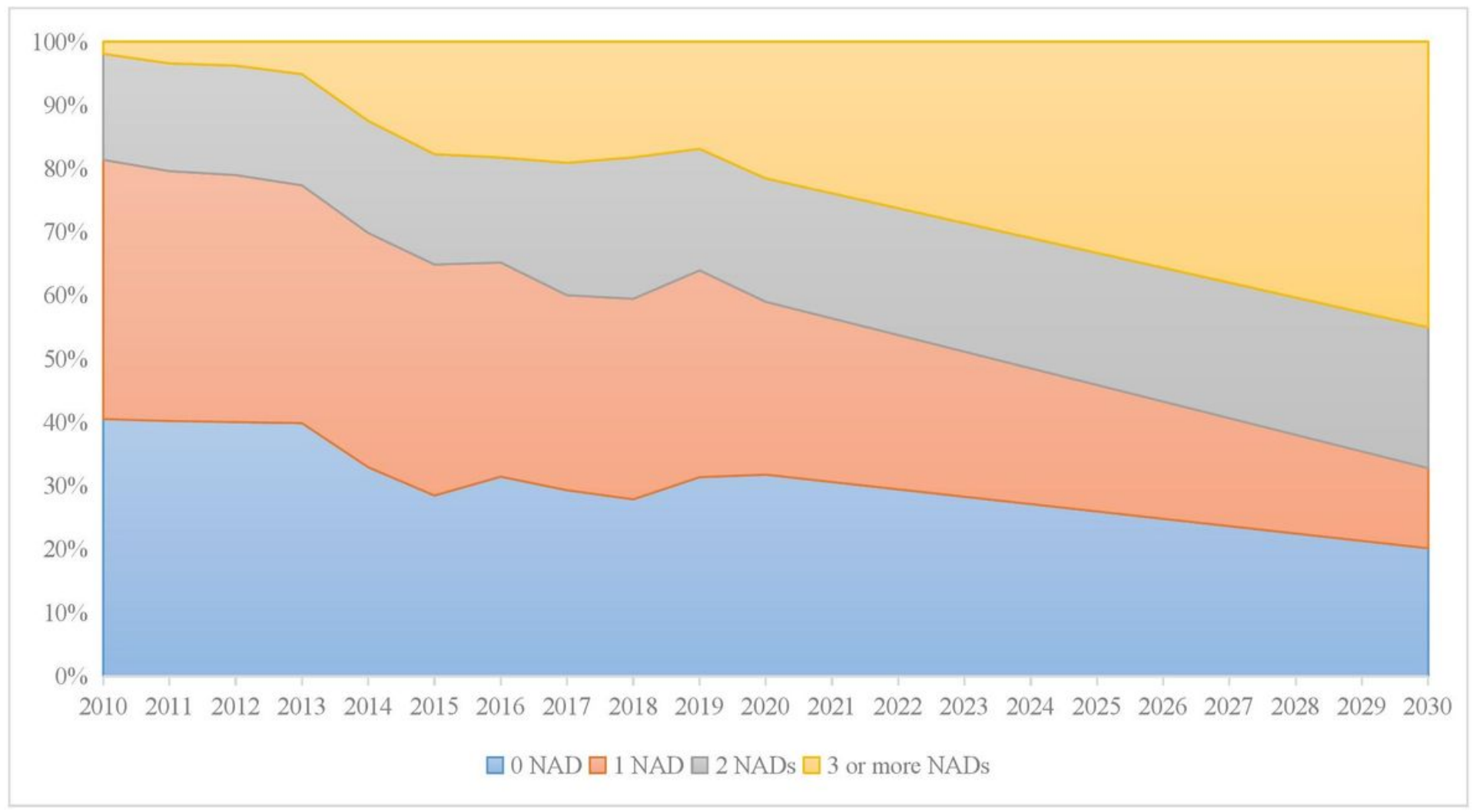

\section{Figure 8}

Changes in proportion of ILWH with multiple NAD

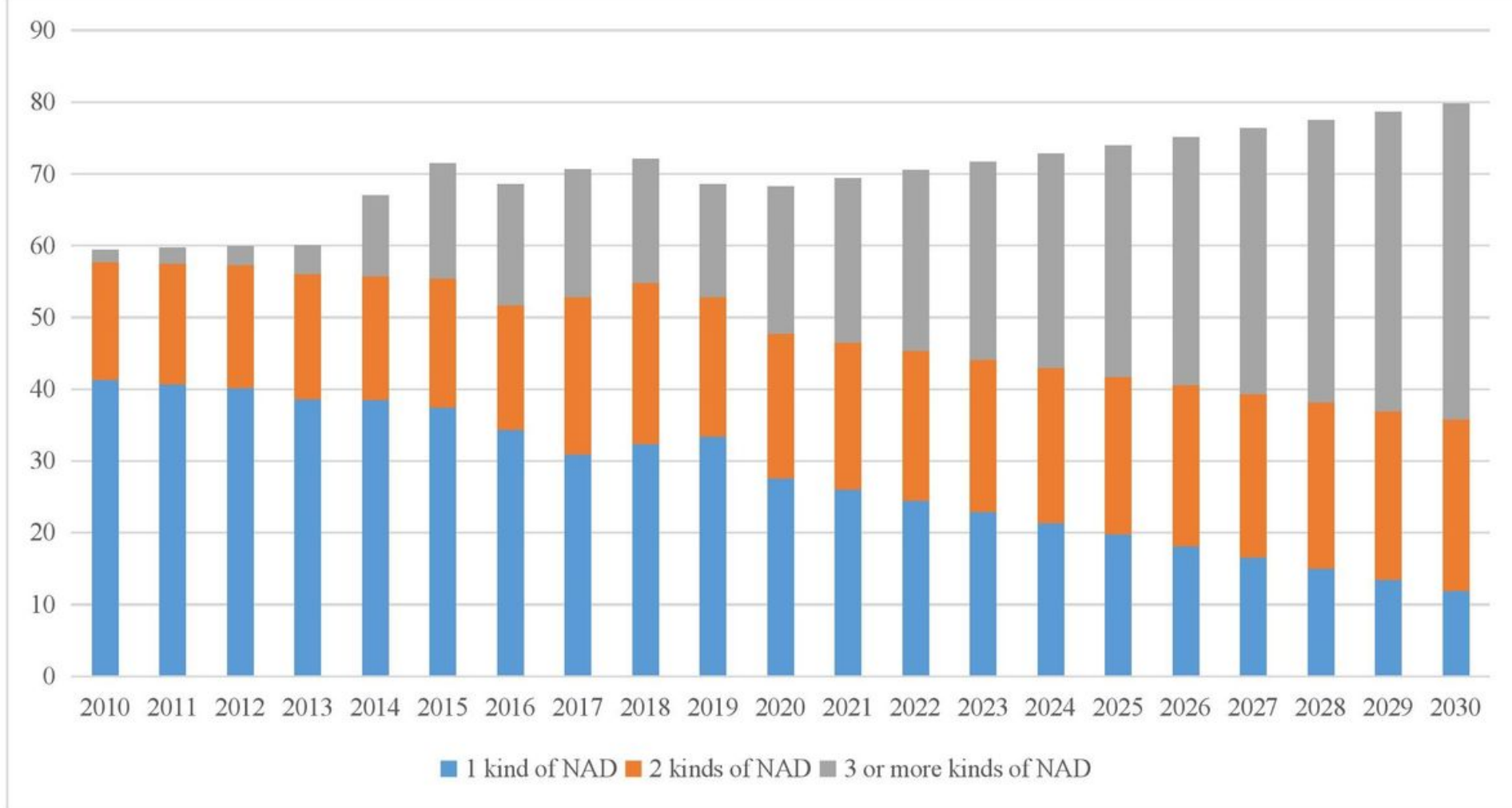

Figure 9 
Changes in proportion of ILWH with multiple types of NAD 